\title{
Hormônio de crescimento e hipersensibilidade por imunocomplexo (Tipo III): uma contraindicação para a dessensibilização
}

\author{
Growth hormone therapy and hypersensitivity type III \\ reaction: a contraindication for desensitization
}

Wilma Carvalho Neves Forte 1,2 , Cristiane Kochi ${ }^{1,2}$, Claudia Dutra Costantin Faria', Luis Eduardo Procópio Calliari', Osmar Monte ${ }^{1,2}$, Carlos Alberto Longui 1,2

1 Irmandade da Santa Casa de Misericórdia de São Paulo (ISCMSP), São Paulo, SP, Brasil ${ }^{2}$ Faculdade de Ciências Médicas da Santa Casa de São Paulo (FCMSCSP), São Paulo, SP, Brasil

Correspondência para: Cristiane Kochi

Rua Dona Veridiana, 311 01238-010

ckochi@uol.com.br

Recebido em 19/Mail/2010 Aceito em 11/Nov/2010

\section{SUMÁRIO}

As reações alérgicas ao GH são raras e usualmente representadas por reações de hipersensibilidade tipo I (IgE mediadas), passíveis de tratamento por dessensibilização. Neste relato de caso, descrevemos a presença de reação alérgica ao GH mediada por imunocomplexo (hipersensibilidade tipo III). Nesta situação, a tentativa de dessensibilização pode perpetuar a formação de imunocomplexo, cujo depósito pode determinar insuficiência renal e respiratória. Arq Bras Endocrinol Metab. 2011;55(1):78-80

\section{SUMMARY}

Allergic reactions against $\mathrm{GH}$ are rare, and usually represented by the hypersensitivity type I (IgE-mediated). This type of reaction can be treated by desensitization. In this case report, we present a patient showing an allergic reaction soon after starting $\mathrm{GH}$ therapy mediated by immune complex (hypersensitivity type III reaction). In this condition, the attempt to perform the desensitization procedure can perpetuate immune complex deposition determining a life threatening renal and respiratory insufficiency. Arq Bras Endocrinol Metab. 2011;55(1):78-80

\section{INTRODUÇÃO}

$\mathrm{R}$ eações alérgicas ao hormônio de crescimento $(\mathrm{GH})$ são raramente descritas, sendo representadas por reações de hipersensibilidade tipo I ou reações $\mathrm{IgE}$ mediadas $(1,2)$. Neste tipo de reação imune, o procedimento de dessensibilização é possível utilizando-se doses muito baixas, progressivamente aumentadas até a dose recomendada (2-5). Para outros hormônios sintéticos, como insulina e hormônio tireoidiano (6), as reações alérgicas mais relatadas também são IgE mediadas.

Ainda mais raras são as reações mediadas por imunocomplexo ou reações de hipersensibilidade tipo III. Neste tipo de reação, a tentativa de dessensibilização pode perpetuar a formação de imunocomplexos, com risco de ocorrer depósito destes, determinando insuficiência renal, respiratória e mesmo óbito.
O presente relato descreve clínica e laboratorialmente uma paciente que durante o uso de $\mathrm{GH}$ apresentou reação alérgica e falha terapêutica, necessitando a distinção entre os tipos I e III de hipersensibilidade.

\section{RELATO DO CASO}

MP, branca, nascida em São Paulo, 12 anos de idade, encaminhada por baixa estatura e obesidade. Criança sempre foi pequena, o que se tornou mais evidente após os 5 anos de idade, quando simultaneamente passou a observar maior ganho ponderal. Apresentava cefaleia holocraniana esporádica e miopia.

Nascida a termo, parto cesário por apresentação pélvica, Apgar 4 e 9, referido como adequada para a idade gestacional. Teve hipoglicemia transitória e icterícia 
neonatal tratada apenas com fototerapia. Alta hospitalar com cinco dias de vida. Apresentou luxação congênita bilateral dos quadris associada a pés tortos congênitos, frouxidão ligamentar dos joelhos e rotação interna da tíbia esquerda. Submetida à imobilização gessada seguida de três correções cirúrgicas dos pés durante os primeiros anos de vida. Entre os 3 e 10 anos de idade, foi submetida a seis procedimentos ortopédicos para correção do quadril e membros inferiores, com resultado cirúrgico satisfatório. Identificado ainda espondilolistese ao nível da vértebra L5, sem necessidade de intervenção cirúrgica.

Antecedentes familiais: mãe com estatura de 158 $\mathrm{cm}$, quatro gestações, um parto, três abortamentos espontâneos relacionados a útero bicorno. Pai com estatura de $173 \mathrm{~cm}$, referindo hipertensão arterial e dislipidemia. Histórico familiar de diabetes melito tipo $2 \mathrm{e}$ hipertensão arterial. Nega consanguinidade.

O exame físico inicial evidenciou peso $=50,7 \mathrm{~kg}(\mathrm{p}$ $=75)$ e estatura de $132,3 \mathrm{~cm}(\mathrm{p}=3)$; com índice de massa corporal de 29,1 (> p95), estágio puberal: M1P1, pressão arterial normal e sem sinais clínicos sugestivos de síndrome de Cushing. Exames laboratoriais gerais normais, idade óssea de 10 anos, cariótipo: 46,XX, CT $=190 \mathrm{mg} / \mathrm{dL} ; \mathrm{HDL}=37 \mathrm{mg} / \mathrm{dL} ; \mathrm{LDL}=131 \mathrm{mg} / \mathrm{dL}$ e triglicérides $=108 \mathrm{mg} / \mathrm{dl}$.

Exames hormonais basais: IGFl $=36 \mathrm{ng} / \mathrm{mL}$ $($ RIE,VR $=110-565) ;$ IGFBP3 $=1,7 \mathrm{mcg} / \mathrm{mL}(\mathrm{RIE}, \mathrm{VR}$ $=1,5-4,3) ; \mathrm{LH}<0,01 \mathrm{mUI} / \mathrm{L}(\mathrm{QL}) ; \mathrm{FSH}=0,4 \mathrm{mUI} /$ $\mathrm{L}(\mathrm{QL}) ; \mathrm{E} 2<10 \mathrm{pg} / \mathrm{mL}(\mathrm{RIE}) ; \mathrm{T} 3=119 \mathrm{ng} / \mathrm{mL} ; \mathrm{T} 4=$ $4,6 \mathrm{mcg} / \mathrm{dL} ; \mathrm{T} 4 \mathrm{~L}=0,66 \mathrm{ng} / \mathrm{dL} ; \mathrm{TSH}=3,3 \mathrm{mUI} / \mathrm{mL}$.

Teste da clonidina: $\mathrm{GH}$ basal $=0,07 \mathrm{ng} / \mathrm{mL}$ e pico $\mathrm{GH}=0,08 \mathrm{ng} / \mathrm{mL}(\mathrm{QL})$.

Teste combinado de avaliação da função hipofisária (Tabela 1) evidenciando falta de resposta do $\mathrm{GH}$ e resposta parcial do cortisol durante hipoglicemia; discreta elevação da prolactina e hiper-resposta do TSH, sugestiva de hipotiroidismo hipotalâmico. Ausência de resposta gonadotrófica.
A ressonância magnética evidenciou intensa hipoplasia de hipófise anterior associada a hipersinal ectópico da hipófise posterior.

A paciente foi medicada com acetato de hidrocortisona $\left(15 \mathrm{mg} / \mathrm{m}^{2}\right)$, dividido em duas doses diárias; levotiroxina sódica ( $100 \mathrm{mcg} / \mathrm{dia})$ e hormônio de crescimento $(0,1 \mathrm{UI} / \mathrm{kg} / \mathrm{dia})$.

Após duas semanas do início da terapia com GH, começou a apresentar lesões de pele, referidas como urticária, além de dor em joelho direito. Ao exame físico, constataram-se lesões máculo-papulares, de diâmetro entre 3 e $4 \mathrm{~cm}$, algumas confluentes, não urticariformes, principalmente em face e tronco; articulações sem sinais flogísticos; adenomegalia cervical, axilar, inguinal e baço palpável ( $1 \mathrm{~cm}$ RCE).

Diante do quadro, foi realizada a dosagem de $\operatorname{IgE}$ total, que se mostrou normal. A pesquisa de imunocomplexos foi realizada em sangue periférico, seguindo-se o método descrito resumidamente a seguir: após coleta de $10 \mathrm{~mL}$ de sangue com material pré-aquecido, este foi imediatamente incubado a $37^{\circ} \mathrm{C}$, separando-se o coágulo após duas horas. O soro foi armazenado a $4^{\circ} \mathrm{C}$ e, após 10 dias, avaliado para constatação de formação do crioprecipitado, que após separação em centrífuga refrigerada foi lavado com soro fisiológico. Realizou-se a solubilização do crioprecipitado após aquecimento. O crioprecipitado foi colocado em placas de imunodifusão radial, identificando-se a formação de halos para $\operatorname{IgM}$, IgG, IGA e componentes C3 e C4 do complemento. Para a identificação de imunocomplexo, o crioprecipitado foi colocado em três ensaios de agarose: um contendo o próprio medicamento utilizado pela paciente $\mathrm{GH}$, outro GH de marca diferente, e um terceiro ensaio contendo apenas o diluente. As amostras do crioprecipitado foram diluídas 1:10 e submetidas ao gel de agarose 1,0\% e $2,4 \%$. A imunodifusão radial do precipitado, mais bem observada após 72 horas, foi somente observada nas placas contendo ambas as apresentações de GH, não havendo imunodifusão na placa contendo apenas o diluente.

Tabela 1. Resultados hormonais durante teste combinado com insulina $(0,1 \mathrm{UI} / \mathrm{kg})$, TRH (75 mcg) e LHRH (100 mcg)

\begin{tabular}{lccccccc}
\hline Tempo & $\begin{array}{c}\text { TSH } \\
\mathbf{m U I / m L}\end{array}$ & PRL & $\begin{array}{c}\text { LH } \\
\mathbf{m U l} / \mathbf{L}\end{array}$ & $\begin{array}{c}\mathbf{F S H} \\
\mathbf{m U I} / \mathbf{L}\end{array}$ & $\begin{array}{c}\mathbf{F} \\
\mathbf{m c g} / \mathbf{d L}\end{array}$ & $\begin{array}{c}\mathbf{G H} \\
\mathbf{n g} / \mathbf{m L}\end{array}$ & $\begin{array}{c}\mathbf{G l i c o s e} \\
\mathbf{m G} / \mathbf{d L}\end{array}$ \\
\hline 0 & 4,2 & 20,6 & 0,8 & 0,5 & 7 & 0,2 & 82 \\
15 & 29,3 & 46,3 & 0,5 & 0,5 & 8,6 & 0,3 & 51 \\
30 & 36,8 & 40,7 & 0,5 & 0,5 & 8,4 & 0,1 & 35 \\
45 & 36,1 & 39,8 & 0,4 & 0,5 & 8,3 & 0,4 & 38 \\
60 & 35,5 & 32,7 & 0,5 & 0,5 & 8,3 & 0,1 & 44 \\
90 & 35,6 & 31,5 & 0,5 & 0,6 & 7,7 & 0,4 & 71 \\
\hline
\end{tabular}


Orientada a suspensão do $\mathrm{GH}$, a regressão dos sinais clínicos de hipersensibilidade foi observada em cerca de 30 dias. Evolutivamente, a paciente foi mantida sem uso de GH, tendo recebido doses substitutivas de levotiroxina, prednisolona e estrogênios conjugados, variando progressivamente de 0,15 a $0,625 \mathrm{mg} /$ dia. Apresentou menarca aos 17,8 anos. Doses atuais de tiroxina $(88 \mathrm{mcg} /$ dia $)$ e prednisolona $(1,5 \mathrm{mg} /$ dia $)$. A evolução pôndero-estatural está na figura 1 .

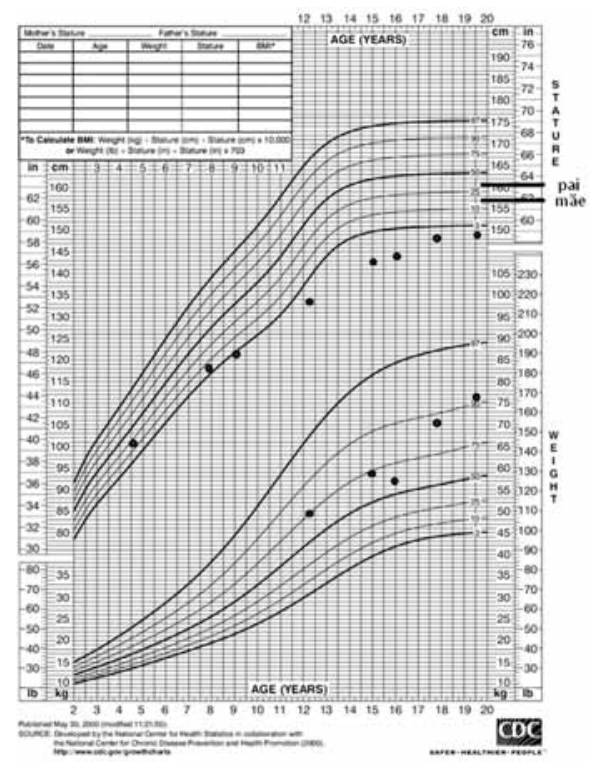

Figura 1. Evolução pôndero-estatural.

\section{DISCUSSÃO}

Os dados observados indicam a presença de hipersensibilidade tipo III (mediada por imunocomplexos). O início dos sintomas após duas semanas de uso do GH reflete o período de latência para o aparecimento de imunocomplexos de tamanho intermediário, ou seja, excesso relativo de moléculas de antígeno em relação ao anticorpo. Os complexos pequenos não desencadeiam a ativação de complemento, enquanto os grandes são rapidamente reconhecidos pelo sistema imunológico e fagocitados. Os imunocomplexos de tamanho intermediário são solúveis, circulando pelo organismo; ao encontrarem barreiras, como sinóvia, alvéolos, glomérulos, depositam-se e a patogenia da doença é determinada pela própria deposição, além da ativação do complemento, determinando lise do local onde houve o depósito. Essa patogenia poderia explicar as alterações cutâneas e a dor articular referida pela paciente, assim como uma possível evolução para insuficiência respiratória e/ou renal apresentada em casos de contínua administração de antígeno, o que não foi o caso do presente relato, pois o GH foi prontamente suspenso. É importante ressaltar que a dessensibilização seria totalmente contraindicada nessa situação, uma vez que não se tratava de reação $\operatorname{IgE}$ mediada.

Laboratorialmente a reação tipo III foi estabelecida pela presença de crioprecipitado. Tivemos o cuidado de afastar a presença de fibrinogênio através da solubilização do crioprecipitado após aquecimento e pela confirmação da presença de $\operatorname{IgM}$, IgG, IgA, componentes C3 e C4 do complemento. A constatação da especificidade do crioprecipitado para GH foi feita através dos ensaios de diluição. Foram tentadas diferentes concentrações e as que permitiram melhor visualização da difusão radial do crioprecipitado da paciente em questão foi a concentração de 1:10 em gel de agarose $1,0 \%$ e 2,4\%. A não formação de halo afasta a possibilidade de formação de anticorpos contra algum contaminante do diluente. A presença de imunodifusão do crioprecipitado por meio de agarose contendo GH de outra marca, bem como contendo o próprio medicamento utilizado pela paciente, constata a especificidade da reação ao hormônio.

Embora ainda não disponível no mercado em nosso país, uma possibilidade terapêutica seria o uso da IGF1 recombinante para o tratamento da baixa estatura.

Concluímos que a paciente apresentou reação de hipersensibilidade tipo III, ou seja, mediada por imunocomplexo, ao hormônio de crescimento utilizado. O presente relato é importante para ressaltar que as reações ao GH não são apenas mediadas por IgE, servindo de alerta para a contraindicação do procedimento de dessensibilização nesse tipo de reação mediada por imunocomplexos.

Declaração: os autores declaram não haver conflitos de interesse científico nesse estudo.

\section{REFERÊNCIAS}

1. Petersen K, Khalaf A, Zeisel H. IgE antibodies to human growth hormone prior to and during the treatment. Acta Endocrinol. 1989;121:501-4.

2. Ranke MB, Bierich JR. Clinical experience with authentic recombinant somatropin. Acta Paediatr Scand. 1987;331:9-17.

3. Walker SB, Weiss ME, Tattoni DS. Systemic reaction to human growth hormone treated with acute desensitization. Pediatrics. 1992;90:108-9.

4. Junprasert J, Javier FC, Rodriguea JA, Moore C, Sorensen RU. Successful intravenous desensitization of growth hormone hypersensitivity. J Ped Endocrinol Metabol. 1997;10:223-6.

5. Davis JRE, Sheppard MC, Shakespear RA, Lynch SS, Clayton RN. Does growth hormone releasing factor desensitize the somatotroph? Interpretation of responses of growth hormone during and after 10-hour infusion of grf 1-29 amide in man. Clin Endocrinol. 2008;24:135-40.

6. Shibata H, Hayakawa H, Hirukawa M. Hypersensitivity caused by synthetic thyroid hormones in a hypothyroid patient with Hashimoto's thyroiditis. Arch Inter Med. 1986;146:1624-5. 